Supporting Information

\title{
Modular Genetic Code Expansion Platform and PISA Yield Well- Defined Protein-Polymer Assemblies
}

Dominic J. Rucco, Brooke E. Barnes, John B. Garrison, Brent S. Sumerlin, Daniel A. Savin*

*George \& Josephine Butler Polymer Research Laboratory, Center for Macromolecular Science \& Engineering, Department of Chemistry, University of Florida, Gainesville, FL 32611, USA.

E-mail: savin@,chem.ufl.edu

Dynamic Light Scattering Results

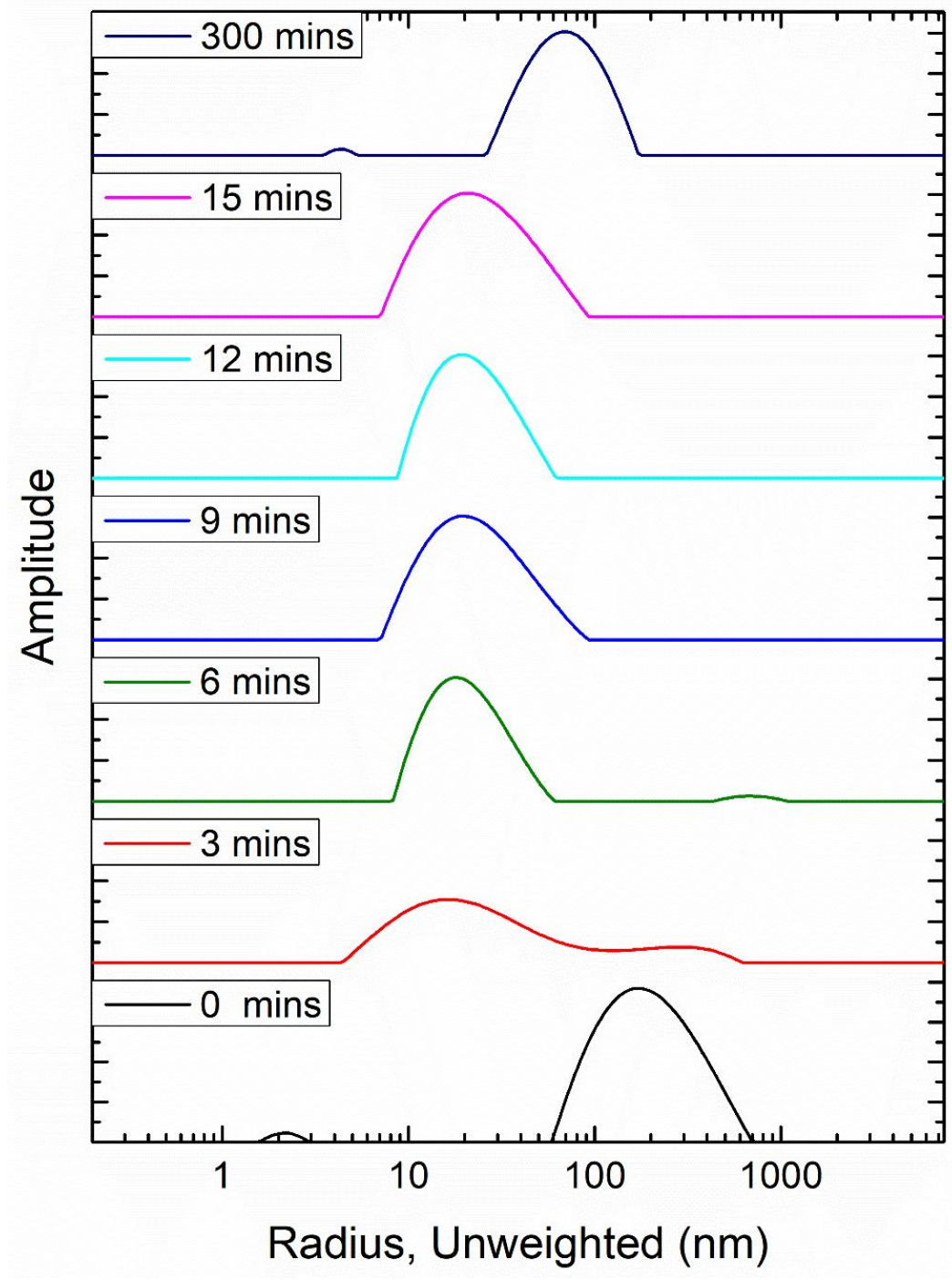


Figure S1. Stacked intensity-weighted CONTIN fits of kinetic data from DLS.

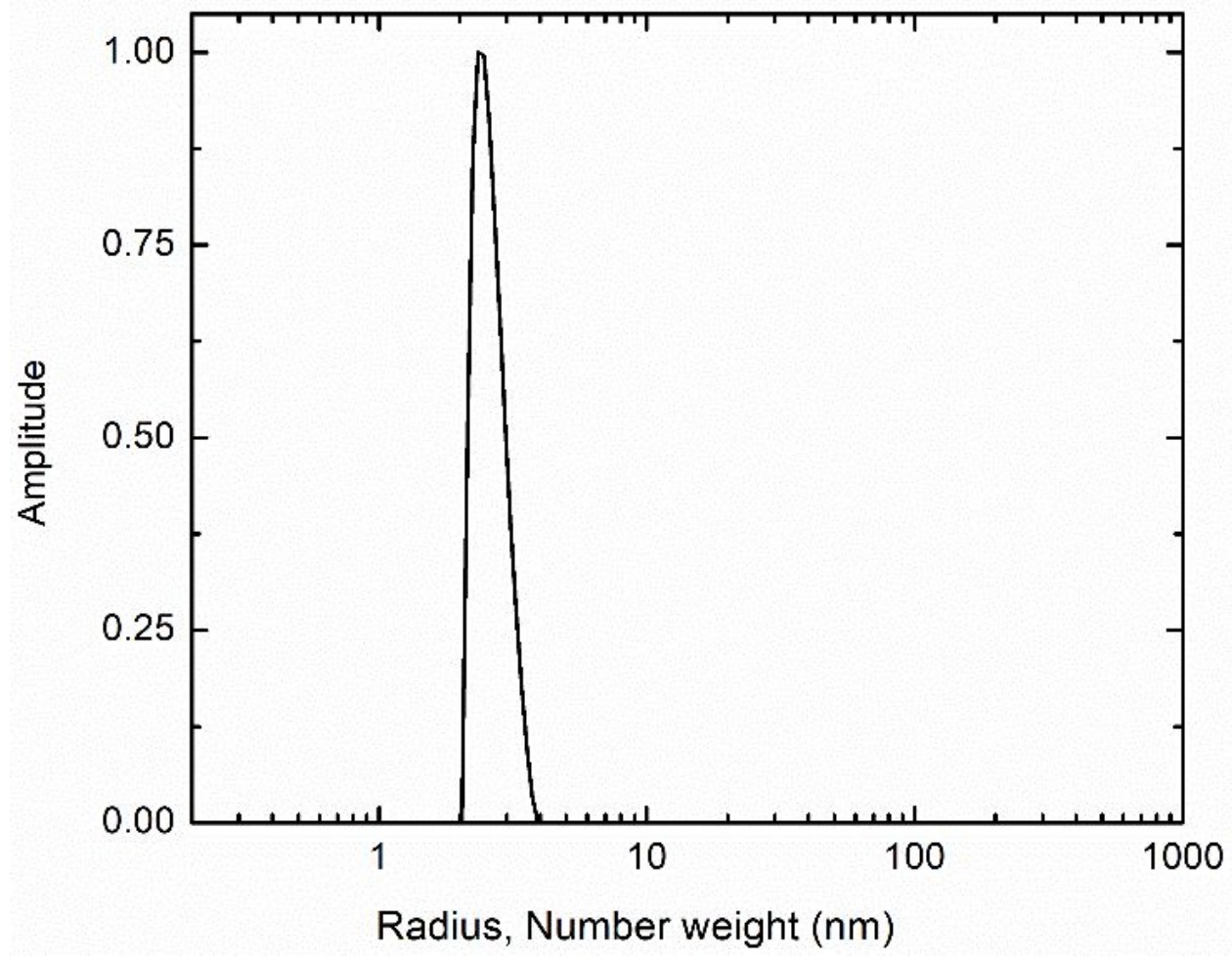

Figure S2. Number-weighted CONTIN fit of starting material ( 0 min) from dynamic light scattering at an angle of $30^{\circ}$. 


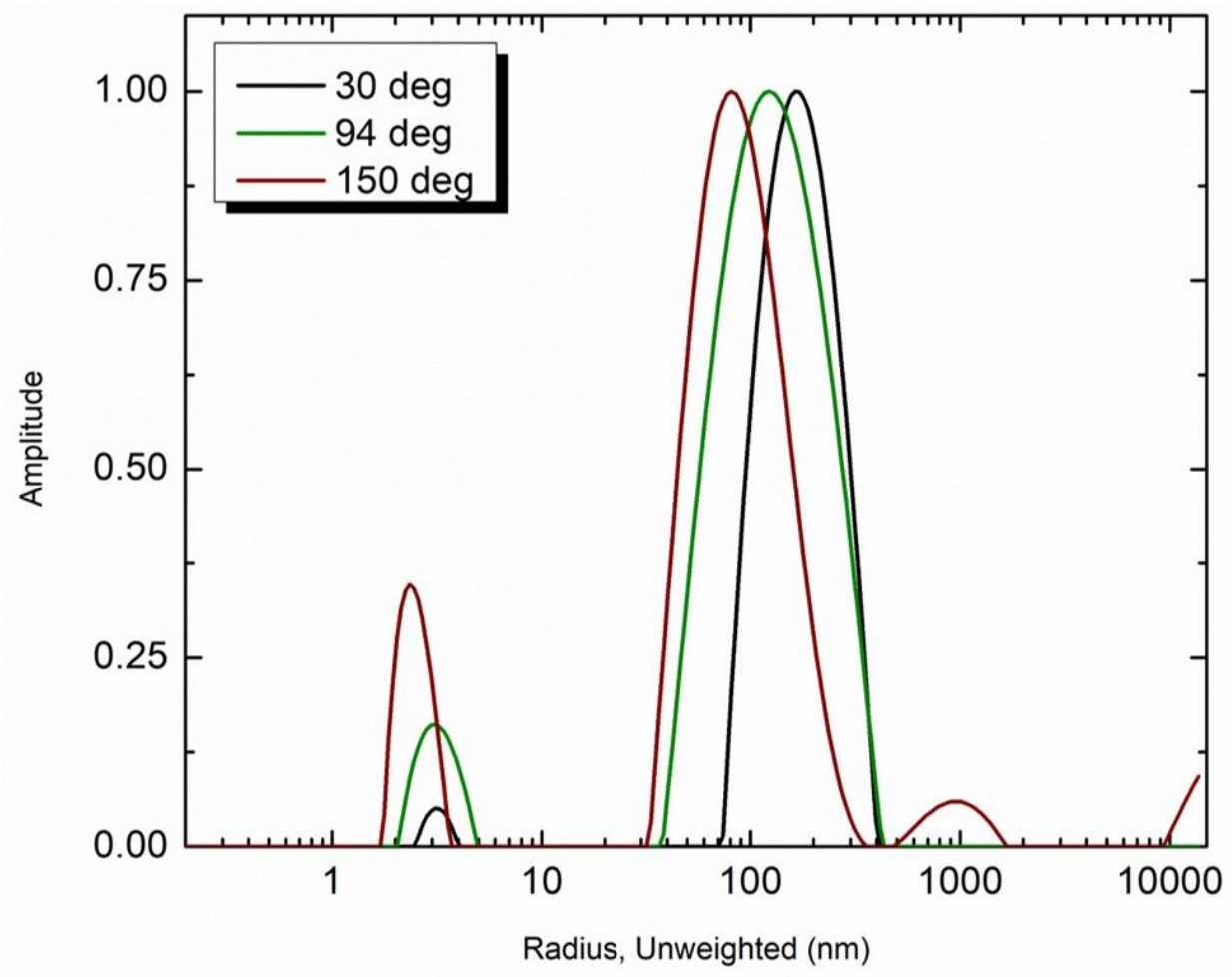

Figure S3. CONTIN fit, intensity-weighted, of starting material ( $0 \mathrm{~min})$ from dynamic light scattering at multiple angles. It is observed that the relative contribution of the larger scattering species decreases at higher angles. This is related to the decrease in the form factor at higher angles, which drops off more steeply for the larger species, resulting in a decreased contribution to the total scattering intensity. 


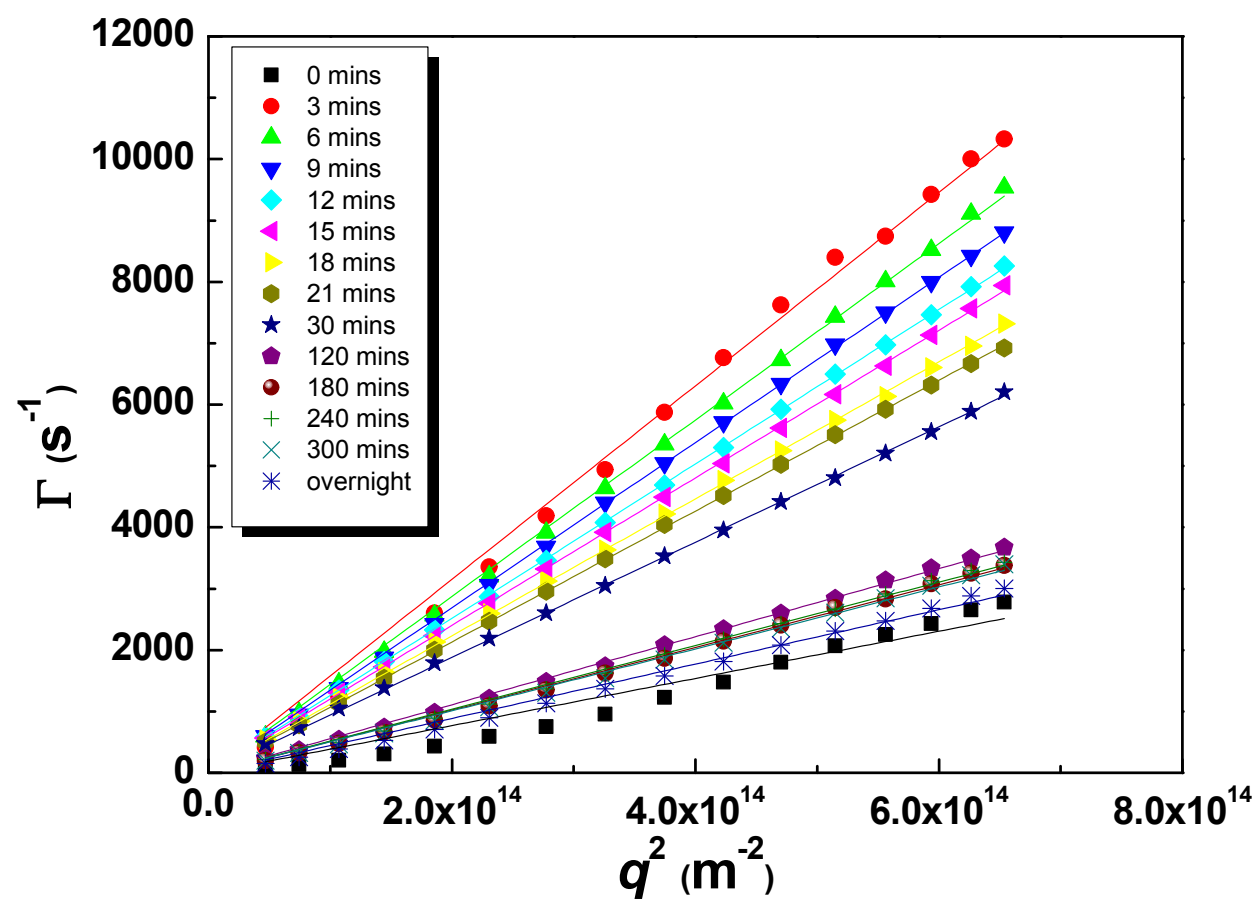

Figure S4. Dynamic light scattering $\Gamma$ vs. $q^{2}$ plot for various polymerization times, showing change in assembly size as a function of polymerization time. Relaxation rate of the correlation function $(\Gamma)$ plotted against the square of the scattering vector $q$ for angles $30^{\circ}-150^{\circ}$. The mutual diffusion coefficient $D_{\mathrm{m}}$ was extracted from the linear slope of each data set. The $\Gamma$ values were calculated by performing a second-order cumulant analysis on the autocorrelation functions. 


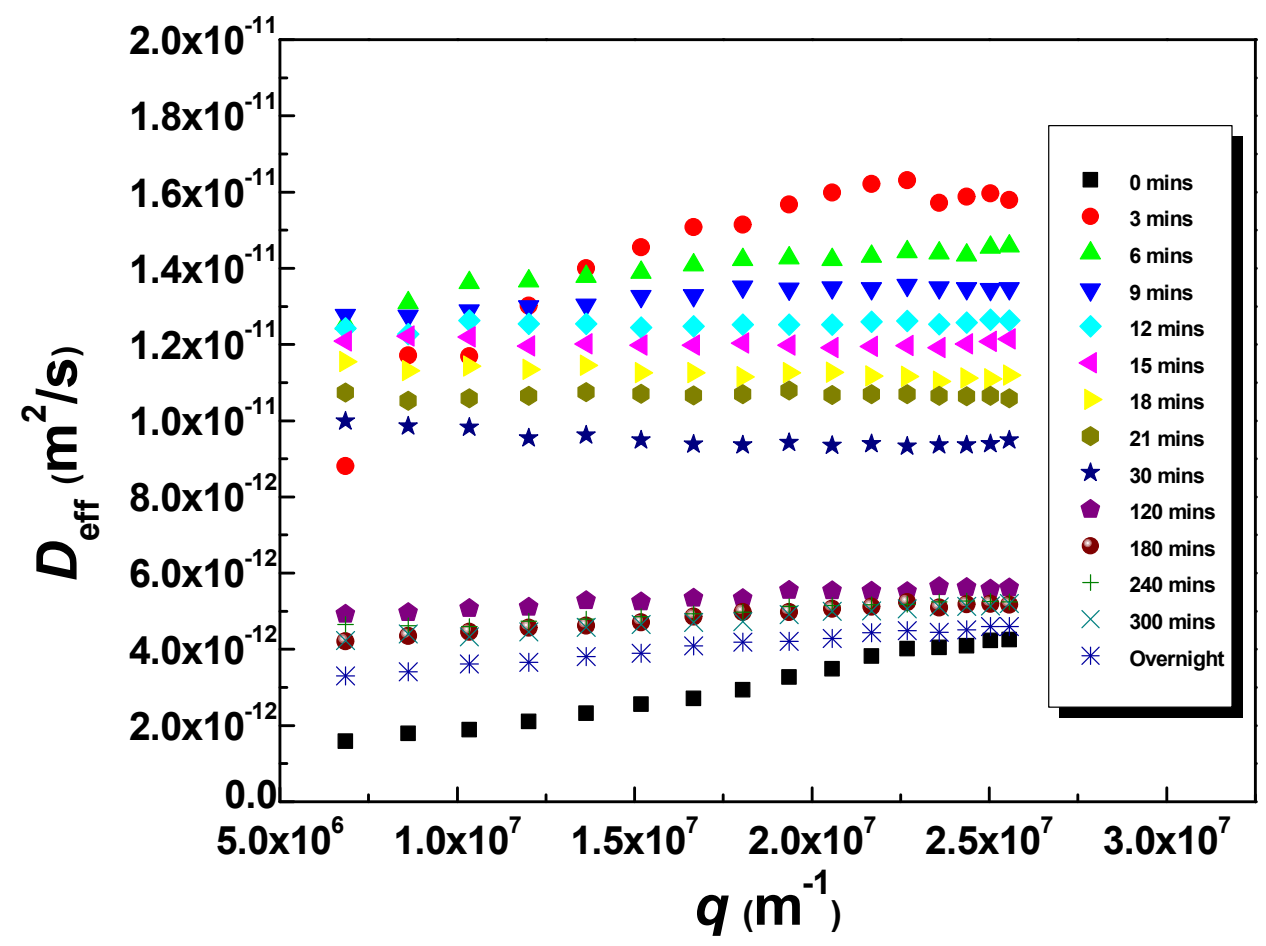

Figure S5. The effective diffusion coefficient $D_{\text {eff }}$ plotted against the scattering vector $q$ for scattering angles $30^{\circ}-150^{\circ}$. A slight upward trend indicates dispersity in the size of the scattering centers, while a zero slope indicates a monodisperse distribution. 


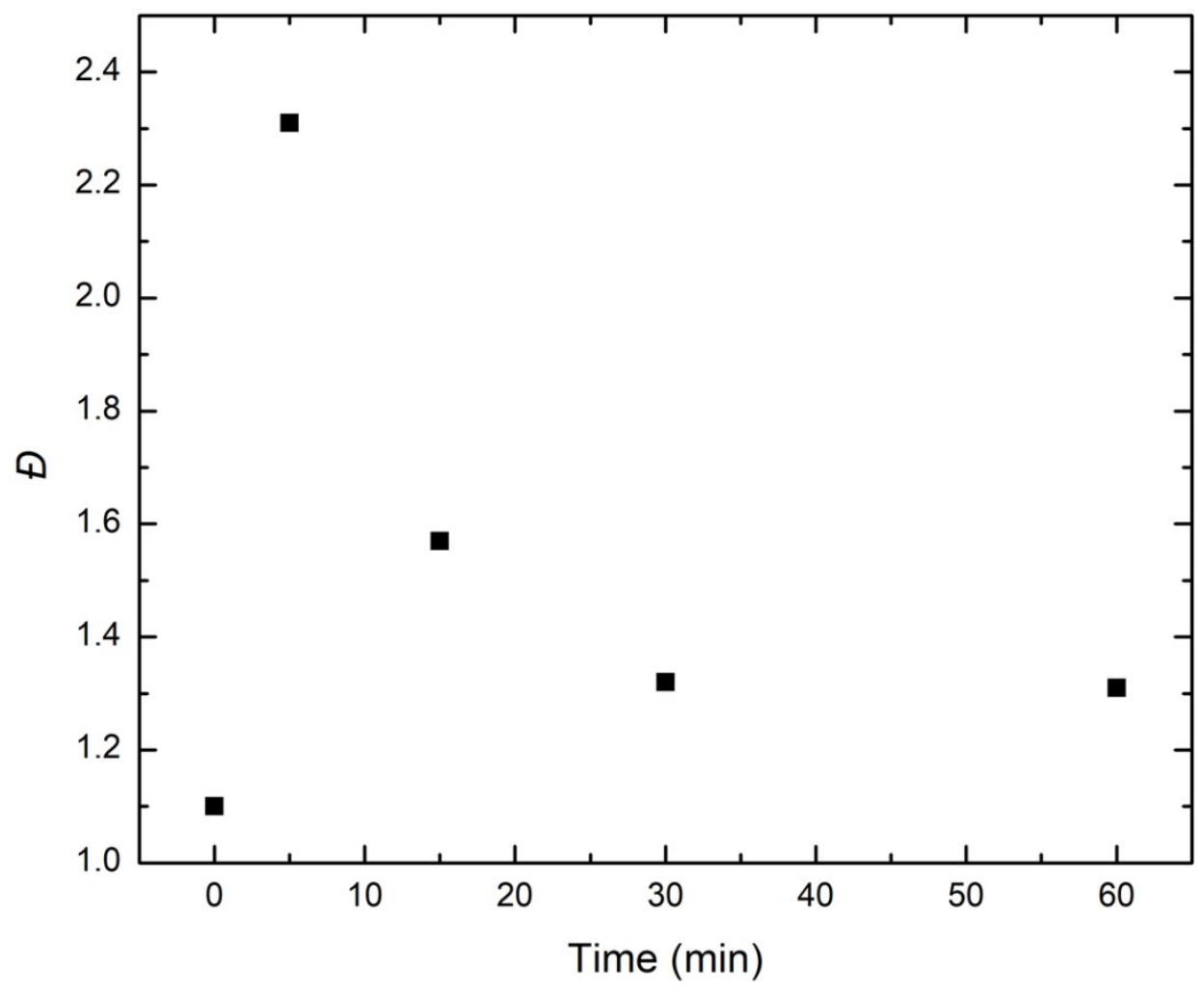

Figure S6. Decreasing bioconjugate molecular weight dispersity by GPC with increasing polymerization time. Values are $1.10,2.31,1.57,1.32$, and 1.31 for $\mathrm{t}=0$ to $60 \mathrm{~min}$, respectively. 